\title{
Predictors of Neurological Deterioration during Admission for Patients with Cerebellar Strokes
}

Thanyalak Amornpojnimman, M.D. ${ }^{1}$, Utcharee Intusoma, M.D. ${ }^{2}$, Nuttha Sanghan, M.D. ${ }^{3}$, Anukoon Kaewborisutsakul, M.D. ${ }^{4}$, Pornchai Sathirapanya, M.D. ${ }^{1}$

'Division of Neurology, Department of Internal Medicine, Faculty of Medicine, Prince of Songkla University, Hat Yai, Songkhla 90110, Thailand.

${ }^{2}$ Division of Neurology, Department of Pediatrics, Faculty of Medicine, Prince of Songkla University, Hat Yai, Songkhla 90110, Thailand.

${ }^{3}$ Division of Neuroradiology, Department of Radiology, Faculty of Medicine, Prince of Songkla University, Hat Yai, Songkhla 90110, Thailand.

${ }^{4}$ Division of Neurological Surgery, Department of Surgery, Faculty of Medicine, Prince of Songkla University, Hat Yai, Songkhla 90110, Thailand.

Received 15 December 2020 • Revised 16 March 2021 • Accepted 17 March 2021 • Published online 2 June 2021

\section{Abstract:}

Objective: Despite less common, cerebellar stroke frequently results in unfavorable outcomes, especially after deterioration. Therefore, this study was aimed to identify the significant predictors of neurological deterioration during admission (NDDA) in ischemic and hemorrhagic cerebellar strokes.

Material and Methods: We retrospectively reviewed all medical records of patients diagnosed with ischemic and hemorrhagic cerebellar strokes; during 2002-2018, in Songklanagarind Hospital. Comparison of patients' demographic data, initial clinical presentations, neuroradiological results, timing and signs of NDDA, and outcomes between cerebellar strokes were descriptively analyzed. Logistic regression model was applied for determining the significant predictors of NDDA from initial clinical presentations.

Results: From this, 74 of 100 patients were eligible. They comprised of $42(57.0 \%)$ cerebellar ischemia $(\mathrm{Cl})$ and 32 (43.0\%) cerebellar hemorrhage $(\mathrm{CH})$. Elevated diastolic blood pressure (DBP) and neuro-radiological evidences suggesting increased posterior cranial fossa pressure were significantly prevalent in neurological deterioration patients. NDDA was

Contact: Pornchai Sathirapanya, M.D.

Division of Neurology, Department of Internal Medicine, Faculty of Medicine, Prince of Songkla University, Hat Yai, Songkhla 90110, Thailand.

E-mail: sporncha@medicine.psu.ac.th
J Health Sci Med Res 2022;40(2):117-128 doi: $10.31584 / j h s m r .2021818$ www.jhsmr.org 
found in 31 patients (42.0\%), without significant difference between $\mathrm{Cl}$ and $\mathrm{CH} .42$ (56.8\%), patients had poor neurological outcomes. The independent predictors for NDDA were DBP $120 \mathrm{mmHg}$ (adjusted odds ratio [adj. OR] 15.39, 95\% Cl 1.58149.59; $p$-value=0.004), time from onset to arrival (adj. OR 0.98, 95\% Cl 0.97-1.00; $p$-value=0.044), and hemispheric cerebellar signs at presentation (adj. OR 0.22, 95\% Cl 0.06-0.75; p-value=0.012).

Conclusion: $\mathrm{CH}$ was not an independent predictor of NDDA in overall cerebellar strokes. Only high DBP predicted NDDA; whereas, time from onset to arrival, and hemispheric cerebellar signs at presentation showed protective impact.

Keywords: cerebellar strokes, during admission, neurological deterioration, predictors

\section{Introduction}

Although, cerebellar stroke is less common (1.0$3.0 \%$ of all strokes), it frequently results in high morbidity and mortality rates (25.0-100\%). ${ }^{1,2}$ Because of the limited space of posterior cranial fossa, where cerebellum locates and the close contact of cerebellum, particularly cerebellar vermis, with the brain stem, ischemic cerebellar edema or cerebellar hematoma poses a high risk of tonsillar herniation as well as direct brain stem compression. These are key mechanisms of secondary neurological deterioration during admission (NDDA) from cerebellar strokes. Generally, NDDA occurs a few days after the onset of cerebellar infarction $(\mathrm{Cl})$, while it commonly takes a few hours after cerebellar hemorrhage $(\mathrm{CH}){ }^{3}$ Therefore, close observation for early detection of neurological deterioration, and timely initiation of neurosurgical interventions are necessary for favorable outcomes after a cerebellar stroke.

There were previous studies reporting the natural course and outcomes of cerebellar strokes. The predictors of poor outcome in cerebellar strokes from several studies included: high blood pressure, high blood glucose level, hydrocephalus and obliterated fourth ventricle from radiologic findings. There have been limited studies reporting the prognosticators of NDDA that required immediate neurosurgical interventions in initially non-surgically indicated patients. ${ }^{1-10}$ Thence, this study was aimed to determine the prognostic factors of surgically-indicated NDDA for both $\mathrm{Cl}$ and $\mathrm{CH}$ from the patient's demographic, and initial presentation characteristics at the emergency department (ED). The findings of our study will be helpful in identify patients at risk, who need intensive monitoring or early neurosurgical intervention.

\section{Material and Methods}

Our primary objective was to identify predictors of secondary neurological deterioration after admission. The secondary objective was to report the neurological outcomes of patients admitted with cerebellar strokes at hospital discharge, and 90 days after onset.

This retrospective cohort study was conducted in Songklanagarind Hospital, an 800-bed tertiary and medical teaching university hospital in southern Thailand. We reviewed the medical records between 1 January 2002 and 31 December 2018.

Medical records of admitted the patients aged $\geq 18$ years who were diagnosed with cerebellar strokes during the study period were reviewed. We excluded patients who had Glasgow coma score (GCS) = 3, or underwent emergency neurosurgical interventions at the time of presentations.

\section{Operational definition of main variables}

The cerebellar strokes were diagnosed by the brain imaging studies, either computed tomography (CT) or magnetic resonance imaging (MRI), and verified by a certified neuroradiologist. 
Midline cerebellar signs were defined by the presence of one, or more truncal ataxia, and a wide based gait.

Hemispheric cerebellar signs were defined by the presence of one, or more impaired finger to nose tests, impaired heel to knee to shin test, dysdiadochokinesia and nystagmus.

Secondary neurological deterioration during admission was defined by the emergence of any new pyramidal tract signs, new brainstem signs, new cerebellar signs or lowering of GCS $>1$ point from the baseline assessment.

The neurological outcomes were evaluated by modified Rankin score (mRs) on the day of hospital discharge, and 90 days after the stroke onset. Stroke outcomes evaluated by $\mathrm{mRs}$ were classified into 2 groups: favorable outcome (mRs 0-2) and unfavorable outcome (mRs 3-6).

Data collected from the electronic medical records included: the patients' demographic data, presenting symptoms and signs, results of routine blood analysis, brain imaging reports, date of NDDA occurrence, and neurosurgical interventions performed for treating NDDA.

Comparison of patient demographic data, initial clinical characteristics at the ED, the presence of NDDA and outcomes between $\mathrm{Cl}$ and $\mathrm{CH}$ were descriptively analyzed. The discrete data were analyzed by chi-square test. The continuous data were analyzed by independent t-test and Mann Whitney $U$ test. The significant variables with $p$-value $<0.200$ in univariate analysis were entered to multivariate logistic regression model. The variables were considered as independent predictors if they meet the statistical significance with a $p$-value $<0.050$ in multivariate logistic regression analysis.

\section{Results}

One hundred cerebellar stroke patients were initially included. After exclusion of twenty-six patients ( 11 cases with $\mathrm{GCS}=3$, and 17 cases with surgical interventions performed at the presentation), 74 non-surgically indicated patients at presentation, admitted for supportive treatments and clinical observation were eligible for final analysis (Figure 1).

There were fifty-two (70.0\%) male and 22 female patients. Among them, 42 patients were $\mathrm{Cl}$ and 32 patients were $\mathrm{CH}$. No significant difference in median (IQR) age was found between the two groups of patients (Table 1). The overall median (IQR) time from stroke onset to hospital arrival was $8(2.12,27.94)$ hours, with significantly shorter time in neurological deterioration patients. Thirteen patients $(5 \mathrm{CH}$ and $8 \mathrm{Cl}$ ) were initially misdiagnosed as peripheral vertigo on their presentation to the emergency department. On evaluation at presentation, we found predominantly high diastolic blood pressure (DBP) in the neurological deterioration group. Significant difference in the presence of cerebellar signs at presentation (midline and hemispheric structure signs) was shown between the two groups. (Table 1) No significant difference in white blood cell count was found between the two groups (Table 1); however, white blood cells $\geq 10,000$ cells $/ \mathrm{mm}^{3}$ were more prevalent in $\mathrm{CH}$ patients. Baseline CT scans and MRI brain were conducted in $70(95.0 \%)$ and $4(5.0 \%)$ cases, respectively. Repeated CT scanning (12 cases) or MRI brain (17 cases) was perfomred to confirm $\mathrm{Cl}$ in initially indefinite CT scan brain reports. The neuro-radiological evidences suggesting increased posterior cranial fossa pressure including obstructive hydrocephalus in 7 (9.5\%), distortion of the fourth ventricle in $6(8.0 \%)$, and tonsillar herniation in $3(4.0 \%)$ cases. Obstructive hydrocephalus and distortion of the fourth ventricle were significantly shown on the brain images of neurological deterioration cases (Table 1).

The overall median (IQR) time from stroke onset to definite diagnosis was $9(3.1,34.2)$ hours. There were $12 / 42(28.6 \%)$ patients with acute $\mathrm{Cl}$ who was diagnosed within 4.5 hours. Notably, no patient received intravenous 
thrombolysis, because of misdiagnosis at presentation as peripheral vertigo, or having contraindications for thrombolytic treatment; such as, high bleeding risk with prolongation of international normalized ratio (INR) and high blood pressure.

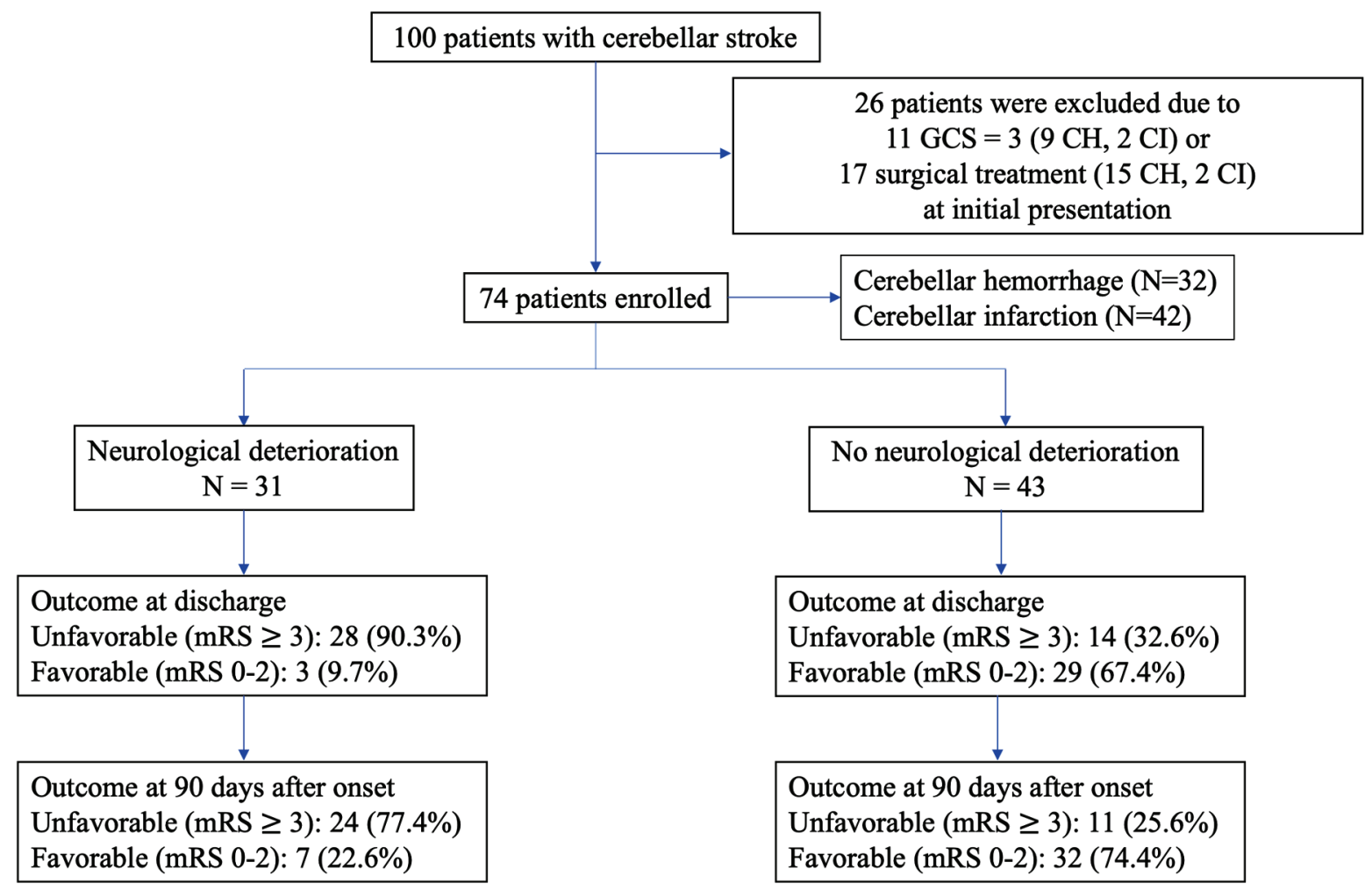

$\mathrm{GCS}=$ Glasgow coma score, $\mathrm{CH}=$ cerebellar hemorrhage, $\mathrm{CI}=$ cerebellar infarction

Figure 1 Study flow 
Table 1 Comparison of patient presentation characteristics between neurological deterioration and no neurological deterioration group during admission in mixed-type cerebellar strokes

\begin{tabular}{|c|c|c|c|}
\hline Variables & $\begin{array}{l}\text { Neurological } \\
\text { deterioration } \\
\text { Number (\%), } \\
n=31\end{array}$ & $\begin{array}{l}\text { No neurological } \\
\text { deterioration } \\
\text { Number }(\%) \text {, } \\
n=43\end{array}$ & p-value \\
\hline Male & $24(77.4)$ & $28(65.1)$ & 0.376 \\
\hline Age, (years) median (IQR) & $67(60.5,74.5)$ & $69(60.0,79.0)$ & 0.288 \\
\hline Time from onset to hospital arrival (hours) median (IQR) & $3(1.0,9.0)$ & $15(5.5,48.0)$ & $0.005^{\#}$ \\
\hline \multicolumn{4}{|l|}{ Risk factors } \\
\hline Hypertension & $19(61.3)$ & $25(58.1)$ & 0.974 \\
\hline Smoking & $11(35.5)$ & $12(27.9)$ & 0.660 \\
\hline Diabetes mellitus & $6(19.4)$ & $11(25.6)$ & 0.728 \\
\hline Dyslipidemia & $8(25.8)$ & $11(25.6)$ & 1.000 \\
\hline Anticoagulant used & $3(9.7)$ & $5(11.6)$ & 1.000 \\
\hline History of CAD & $6(19.4)$ & $3(7.0)$ & 0.153 \\
\hline \multicolumn{4}{|l|}{ Clinical presentation } \\
\hline Nausea or vomiting & $20(64.5)$ & $31(72.1)$ & 0.660 \\
\hline Vertigo or dizziness & $17(54.8)$ & $33(76.7)$ & 0.083 \\
\hline Headache & $17(54.8)$ & $19(44.2)$ & 0.504 \\
\hline Gait ataxia & $10(32.3)$ & $18(41.9)$ & 0.550 \\
\hline Dysarthria & $9(29.0)$ & $10(23.3)$ & 0.771 \\
\hline Limb ataxia & $8(25.8)$ & $7(16.3)$ & 0.476 \\
\hline Alteration of consciousness & $4(12.9)$ & $1(2.3)$ & 0.154 \\
\hline Diplopia & $0(0.0)$ & $2(4.7)$ & 0.506 \\
\hline Tinnitus & $1(3.2)$ & $1(2.3)$ & 1.000 \\
\hline \multicolumn{4}{|l|}{ Initial clinical signs; } \\
\hline $\mathrm{SBP} \geq 180 \mathrm{mmHg}$ & $17(54.8)$ & $13(30.2)$ & 0.059 \\
\hline $\mathrm{DBP} \geq 120 \mathrm{mmHg}$ & $10(32.3)$ & $1(2.3)$ & $<0.001^{d}$ \\
\hline Glasgow coma scale (GCS) & & & 0.069 \\
\hline GCS 13 & $27(87.1)$ & $42(97.7)$ & \\
\hline GCS 9-12 & $1(3.2)$ & $1(2.3)$ & \\
\hline GCS 4-8 & $3(9.7)$ & $0(0.0)$ & \\
\hline \multicolumn{4}{|l|}{ Cerebellar signs at presentation } \\
\hline Midline structure signs & $13(41.9)$ & $30(69.8)$ & $0.031^{\dagger}$ \\
\hline Hemispheric signs & $16(51.6)$ & $34(79.1)$ & $0.025^{\dagger}$ \\
\hline \multicolumn{4}{|l|}{ Initial laboratory finding } \\
\hline WBC $\geq 10,000$ cells $/ \mathrm{mm}^{3}$ & $21(67.7)$ & $19(44.2)$ & 0.077 \\
\hline INR $\geq 1.5$ & $2(6.9)$ & $3(11.1)$ & 0.664 \\
\hline $\begin{array}{l}\text { Blood sugar } 140 \mathrm{mg} / \mathrm{dL} \\
\text { Type of cerebellar stroke }\end{array}$ & $12(38.7)$ & $9(20.9)$ & 0.158 \\
\hline \multicolumn{4}{|c|}{ Initial neuro-imaging results showing increased posterior fossa pressure } \\
\hline Obstructive hydrocephalus & $7(22.6)$ & $0(0.0)$ & $0.001^{d}$ \\
\hline $4^{\text {th }}$ ventricular distortion & $6(19.4)$ & $0(0.0)$ & $0.004^{d}$ \\
\hline Tonsillar herniation & $3(9.7)$ & $0(0.0)$ & 0.069 \\
\hline Vermis involvement & $9(29.0)$ & $15(34.9)$ & 0.780 \\
\hline
\end{tabular}


Table 1 (continued)

\begin{tabular}{|c|c|c|c|}
\hline Variables & $\begin{array}{l}\text { Neurological } \\
\text { deterioration } \\
\text { Number (\%), } \\
n=31\end{array}$ & $\begin{array}{l}\text { No neurological } \\
\text { deterioration } \\
\text { Number }(\%) \text {, } \\
n=43\end{array}$ & p-value \\
\hline \multicolumn{4}{|c|}{ Deterioration during admission $(n=31)$} \\
\hline \multicolumn{4}{|l|}{ Signs } \\
\hline GCS dropped >1 & $20(64.5)$ & & \\
\hline New cerebellar sign & $8(25.8)$ & & \\
\hline New pyramidal sign & $6(19.4)$ & & \\
\hline New brainstem sign & $6(19.4)$ & & \\
\hline \multicolumn{4}{|l|}{ Treatment } \\
\hline Surgical treatment $(n=16)$ & $16(51.6)$ & $0(0.0)$ & $<0.001^{\dagger}$ \\
\hline Type of surgery & & & $<0.001^{\dagger}$ \\
\hline Ventriculostomy & $5(31.2)$ & $0(0.0)$ & \\
\hline Suboccipital craniectomy & $6(37.5)$ & $0(0.0)$ & \\
\hline Both & $5(31.2)$ & $0(0.0)$ & \\
\hline \multicolumn{4}{|l|}{ Outcome } \\
\hline mRS at discharge & & & $<0.001^{\dagger}$ \\
\hline 0-2 (non-dependency) & $3(9.7)$ & $29(67.4)$ & \\
\hline 3-6 (dependency) & $28(90.3)$ & $14(32.6)$ & \\
\hline $\mathrm{mRS}$ at 90 days after onset & & & $<0.001^{\dagger}$ \\
\hline 0-2 (non-dependency) & $7(22.6)$ & $32(74.4)$ & \\
\hline 3-6 (dependency) & $24(77.4)$ & $11(25.6)$ & \\
\hline
\end{tabular}

$\mathrm{CAD}=$ coronary artery disease, $\mathrm{SBP}=$ systolic blood pressure, $\mathrm{DBP}=$ diastolic blood pressure, $\mathrm{GCS}=\mathrm{Glasgow}$ coma score, Midline structure signs=one or more of truncal ataxia and wide based gait, Hemispheric signs=one or more of impaired finger to nose test, impaired heel to knee to shin test, dysdiadochokinesia and nystagmus $\mathrm{WBC}=$ white blood cell, INR=international normalized ratio, mRS=modified Rankin scale, \#=Rank sum test $p$-value $<0.050, f=$ Chi square test $p$-value $<0.050$, $d=$ Fisher's exact test $p$-value $<0.050, I Q R=$ interquartile range

NDDA developed in $31(42.0 \%)$ patients, in whom decreasing of GCS $>1$ point was the most common, alarming neurological sign: this finding was common in $\mathrm{CH}$ patients. The median (IQR) time from onset to NDDA was significantly shorter in $\mathrm{CH}(22(5,48)$ vs. $57(27.5,106)$; p-value=0.031) (Table 2). At the time of NDDA, neurosurgical interventions were performed in $16(51.6 \%)$ cases: 6 (37.5\%) either with decompression craniectomy, 5 (31.3\%) with ventriculostomy, and a combination of both in $5(31.3 \%)$ patients. The rest of the patients, who were indicated for neurosurgical treatment, did not consent for the interventions.

Subsequently, the significant independent predictor for NDDA was DBP $120 \mathrm{mmHg}$ (adjusted OR [adj. OR]
15.39, 95\% Cl 1.58-149.59; p-value=0.004). Notably, time from onset to hospital arrival (adj. OR 0.98, 95\% Cl 0.97-1; $\mathrm{p}$-value=0.044), and hemispheric cerebellar signs at presentation (adj. OR 0.22, 95\% Cl 0.06-0.75; p-value=0.012) were significant protective factors of NDDA (Table 3).

The mean ( \pm standard deviation, S.D.) hospital stay was $17.0 \pm 29.8$ days. The overall outcomes were 42 (56.8\%) patients had unfavorable neurological outcomes, in which $8(10.8 \%)$ patients were deceased at time of discharge. Thirty-five (47.3\%) patients had unfavorable outcomes at 90 days after onset. $\mathrm{Cl}$ had better outcome evaluated by $\mathrm{mRS}$ on the discharge date, but no difference in the outcome at 90 days after onset was found (Table 2). 
Table 2 Comparison of patient presentation characteristics between hemorrhagic and ischemic cerebellar stroke

\begin{tabular}{|c|c|c|c|}
\hline Variables & $\begin{array}{l}\text { Hemorrhage } \\
\text { Number (\%), } \\
n=32\end{array}$ & $\begin{array}{l}\text { Ischemia } \\
\text { Number (\%), } \\
\mathrm{n}=42\end{array}$ & p-value \\
\hline Male & $25(78.1)$ & $27(64.3)$ & 0.301 \\
\hline Age, (years) median (IQR) & $67.5(60.2,79.3)$ & $67(60.25,75.8)$ & 0.658 \\
\hline Time from onset to hospital arrival (hours) median (IQR) & $5.5(1.0,13.2)$ & $13.5(3.0,48.0)$ & $0.035^{\#}$ \\
\hline Misdiagnosis as peripheral vestibular disorders at presentation & $5(15.6)$ & $8(19.0)$ & 0.940 \\
\hline \multicolumn{4}{|l|}{ Risk factors } \\
\hline Hypertension & $17(53.1)$ & $27(64.3)$ & 0.466 \\
\hline Smoking & $7(21.9)$ & $16(38.1)$ & 0.215 \\
\hline Diabetes mellitus & $4(12.5)$ & $13(31.0)$ & 0.112 \\
\hline Dyslipidemia & $6(18.8)$ & $13(31.0)$ & 0.357 \\
\hline Anticoagulant used & $5(15.6)$ & $3(7.1)$ & 0.280 \\
\hline History of CAD & $4(12.5)$ & $5(11.9)$ & 1.000 \\
\hline \multicolumn{4}{|l|}{ Clinical presentation } \\
\hline Nausea or vomiting & $22(68.8)$ & $29(69.0)$ & 1.000 \\
\hline Vertigo or dizziness & $19(59.4)$ & $31(73.8)$ & 0.288 \\
\hline Headache & $23(71.9)$ & $13(31.0)$ & $0.001^{\dagger}$ \\
\hline Gait ataxia & $8(25.0)$ & $20(47.6)$ & 0.081 \\
\hline Dysarthria & $9(28.1)$ & $10(23.8)$ & 0.879 \\
\hline Limb ataxia & $3(9.4)$ & $12(28.6)$ & 0.081 \\
\hline Alteration of consciousness & $3(9.4)$ & $2(4.8)$ & 0.647 \\
\hline Diplopia & $1(3.1)$ & $1(2.4)$ & 1.000 \\
\hline Tinnitus & $1(3.1)$ & $1(2.4)$ & 1.000 \\
\hline \multicolumn{4}{|l|}{ Initial clinical signs; } \\
\hline $\mathrm{SBP} \geq 180 \mathrm{mmHg}$ & $17(53.1)$ & $13(31.0)$ & 0.092 \\
\hline $\mathrm{DBP} \geq 120 \mathrm{mmHg}$ & $8(25.0)$ & $3(7.1)$ & $0.048^{d}$ \\
\hline Glasgow coma scale (GCS) & & & $0.039^{d}$ \\
\hline GCS $\geq 13$ & $29(90.6)$ & $40(95.2)$ & \\
\hline GCS 9-12 & $0(0.0)$ & $2(4.8)$ & \\
\hline GCS 4-8 & $3(9.4)$ & $0(0.0)$ & \\
\hline \multicolumn{4}{|l|}{ Cerebellar signs at presentation } \\
\hline Midline structure signs & $16(50.0)$ & $27(64.3)$ & 0.319 \\
\hline Hemispheric signs & $23(71.9)$ & $27(64.3)$ & 0.660 \\
\hline \multicolumn{4}{|l|}{ Initial laboratory finding } \\
\hline WBC $\geq 10,000$ cells $/ \mathrm{mm}^{3}$ & $22(68.8)$ & $18(42.9)$ & $0.048^{\dagger}$ \\
\hline INR $\geq 1.5$ & $4(13.3)$ & $1(3.8)$ & 0.358 \\
\hline Blood sugar $\geq 140 \mathrm{mg} / \mathrm{dL}$ & $12(37.5)$ & $9(21.4)$ & 0.208 \\
\hline \multicolumn{4}{|c|}{ Initial neuro-imaging results showing increased posterior fossa pressure } \\
\hline Obstructive hydrocephalus & $6(18.8)$ & $1(2.4)$ & $0.038^{\dagger}$ \\
\hline $4^{\text {th }}$ ventricular distortion & $6(18.8)$ & $0(0.0)$ & $0.005^{\dagger}$ \\
\hline Tonsillar herniation & $2(6.2)$ & $1(2.4)$ & 0.575 \\
\hline Vermis involvement & $9(28.1)$ & $15(35.7)$ & 0.660 \\
\hline \multicolumn{4}{|l|}{ Deterioration during admission } \\
\hline Neurological deterioration & $17(53.1)$ & $14(33.3)$ & 0.141 \\
\hline Time from onset to deterioration (hours): median (IQR) & $22(5.0,48.0)$ & $57(27.5,106.0)$ & $0.031^{\#}$ \\
\hline \multicolumn{4}{|l|}{ Signs } \\
\hline GCS dropped >1 & $13(40.6)$ & $7(16.7)$ & $0.042^{f}$ \\
\hline New cerebellar sign & $2(6.2)$ & $6(14.3)$ & 0.453 \\
\hline New pyramidal sign & $4(12.5)$ & $2(4.8)$ & 0.393 \\
\hline New brainstem sign & $4(12.5)$ & $2(4.8)$ & 0.393 \\
\hline
\end{tabular}


Table 2 (continued)

\begin{tabular}{|c|c|c|c|}
\hline Variables & $\begin{array}{l}\text { Hemorrhage } \\
\text { Number (\%), } \\
n=32\end{array}$ & $\begin{array}{l}\text { Ischemia } \\
\text { Number (\%), } \\
n=42\end{array}$ & $p$-value \\
\hline \multicolumn{4}{|l|}{ Treatment } \\
\hline Surgical treatment $(n=16)$ & $8(25.0)$ & $8(19.0)$ & 0.740 \\
\hline Type of surgery & & & 0.283 \\
\hline Ventriculostomy & $4(50.0)$ & $1(12.5)$ & \\
\hline Suboccipital craniectomy & $3(37.5)$ & $3(37.5)$ & \\
\hline Both & $1(12.5)$ & $4(50.0)$ & \\
\hline \multicolumn{4}{|l|}{ Outcome } \\
\hline $\mathrm{mRS}$ at discharge & & & $0.040^{f}$ \\
\hline 0-2 (non-dependency) & $9(28.1)$ & $23(54.8)$ & \\
\hline 3-6 (dependency) & $23(71.9)$ & $19(45.2)$ & \\
\hline $\mathrm{mRS}$ at 90 days after onset & & & 0.266 \\
\hline 0-2 (non-dependency) & $14(43.8)$ & 25 (59.5) & \\
\hline 3-6 (dependency) & $18(56.2)$ & $17(40.5)$ & \\
\hline
\end{tabular}

$\mathrm{CAD}=$ coronary artery disease, $\mathrm{SBP}=$ systolic blood pressure, $\mathrm{DBP}=$ diastolic blood pressure, $\mathrm{GCS}=$ Glasgow coma score, Midline structure signs=one or more of truncal ataxia and wide based gait, Hemispheric signs=one or more of impaired finger to nose test, impaired heel to knee to shin test, dysdiadochokinesia and nystagmus $\mathrm{WBC}=$ white blood cell, INR=international normalized ratio, mRS=modified Rankin scale, \#=Rank sum test $p$-value $<0.050, f=$ Chi square test $p$-value $<0.050, d=$ Fisher's exact test $p$-value $<0.050, I Q R=$ interquartile range

Table 3 Factors associated with neurological deterioration during admission in mixed-type cerebellar strokes

\begin{tabular}{|c|c|c|c|c|}
\hline \multirow[b]{2}{*}{ Variables } & \multicolumn{2}{|c|}{ Univariate analysis } & \multicolumn{2}{|c|}{ Multivariate analysis } \\
\hline & $\begin{array}{l}\text { Crude OR } \\
(95 \% \mathrm{Cl})\end{array}$ & p-value* & $\begin{array}{l}\text { Adjusted OR } \\
(95 \% \mathrm{Cl})\end{array}$ & $p$-value \\
\hline Hemorrhagic stroke (ref=ischemic) & $2.27(0.88-5.83)$ & 0.141 & $1.43(0.40-5.17)$ & 0.586 \\
\hline Previous history of CAD (ref=none) & $3.20(0.73-13.96)$ & 0.153 & $4.04(0.76-21.51)$ & 0.139 \\
\hline Time from onset to hospital arrival (hours) & $0.98(0.96-1.00)$ & 0.005 & $0.98(0.97-1.00)$ & $0.044^{\star \star}$ \\
\hline \multicolumn{5}{|l|}{ Main presenting symptoms before arrival } \\
\hline Dizziness/vertigo (ref=none) & $2.72(1.00-7.39)$ & 0.083 & $0.99(0.25-3.92)$ & 0.986 \\
\hline Alteration of consciousness (ref=none) & & 0.154 & & \\
\hline \multicolumn{5}{|l|}{ Clinical profiles at hospital arrival } \\
\hline $\mathrm{SBP} \geq 180 \mathrm{mmHg}($ ref< $<180 \mathrm{mmHg})$ & $2.80(1.07-7.33)$ & 0.059 & $0.68(0.18-2.56)$ & 0.564 \\
\hline $\mathrm{DBP} \geq 120 \mathrm{mmHg}($ ref $<120 \mathrm{mmHg})$ & $20.00(2.40-166.77)$ & $<0.001$ & $15.39(1.58-149.59)$ & $0.004^{* *}$ \\
\hline GCS $<13($ ref $\geq 13)$ & & 0.154 & & \\
\hline Midline structure signs (ref=none) & & 0.031 & & \\
\hline Hemispheric cerebellar signs (ref=none) & $0.28(0.10-0.78)$ & 0.025 & $0.22(0.06-0.75)$ & $0.012^{\star \star}$ \\
\hline WBC $\geq 10,000$ cells $/ \mathrm{mm}^{3}($ ref $<10,000)$ & $2.65(1.01-6.96)$ & 0.077 & $3.06(0.94-10.02)$ & 0.148 \\
\hline $\mathrm{BS} \geq 140 \mathrm{mg} / \mathrm{dL} \quad(\mathrm{ref}<140 \mathrm{mg} / \mathrm{dL})$ & $2.39(0.85-6.69)$ & 0.158 & $1.54(0.39-5.97)$ & 0.538 \\
\hline \multicolumn{5}{|l|}{ Neuro-imaging finding } \\
\hline Hydrocephalus (ref=none) & & 0.001 & & \\
\hline Tonsillar herniation (ref=none) & & 0.069 & & \\
\hline $4^{\text {th }}$ ventricular distortion (ref=none) & & 0.004 & & \\
\hline
\end{tabular}

$\mathrm{CAD}=$ coronary artery disease, $\mathrm{SBP}=$ systolic blood pressure, $\mathrm{DBP}=$ diastolic blood pressure, $\mathrm{GCS}=\mathrm{Glasgow}$ coma score, Midline structure signs=one or more of truncal ataxia and wide based gait, Hemispheric signs=one or more of impaired finger to nose test, impaired heel to knee to shin test, dysdiadochokinesia and nystagmus, WBC=white blood cell, BS=blood sugar, $\mathrm{mmHg}=$ millimetres mercury, ref=reference, OR=odd ratio

${ }^{*} \mathrm{p}$-value $<0.200,{ }^{* *} \mathrm{p}$-value $<0.050$ adjusted with all variables shown in the table 
In comparison of the number of cases with unfavorable outcomes between patients, with and without NDDA, at the discharge date and 90 days after onset, there were $28(90.0 \%)$ and $24(77.0 \%)$ cases in NDDA group compared to $14(33.0 \%)$ and $11(26.0 \%)$ cases in non-NDDA patients, respectively (Figure 1). We also found that NDDA cerebellar stroke patients had a significant proportion of unfavorable outcomes evaluated by $\mathrm{mRs}$ at hospital discharge $(p$-value $<0.001)$ and at 90 days after onset ( $p$-value<0.001) (Figure 2, 3).

\section{Discussion}

Our study revealed that DBP $120 \mathrm{mmHg}$ was an independent predictor of NDDA (adj. OR 15.39, 95\% Cl 1.58-149.59; $p$-value=0.004) (Table 3). Elevation of blood pressure has been considered as a response to elevation of ICP at stroke onset; however, it probably leads to neurological deterioration because of increased risk of massive cerebral edema and hematoma expansion as well. $^{11-16}$

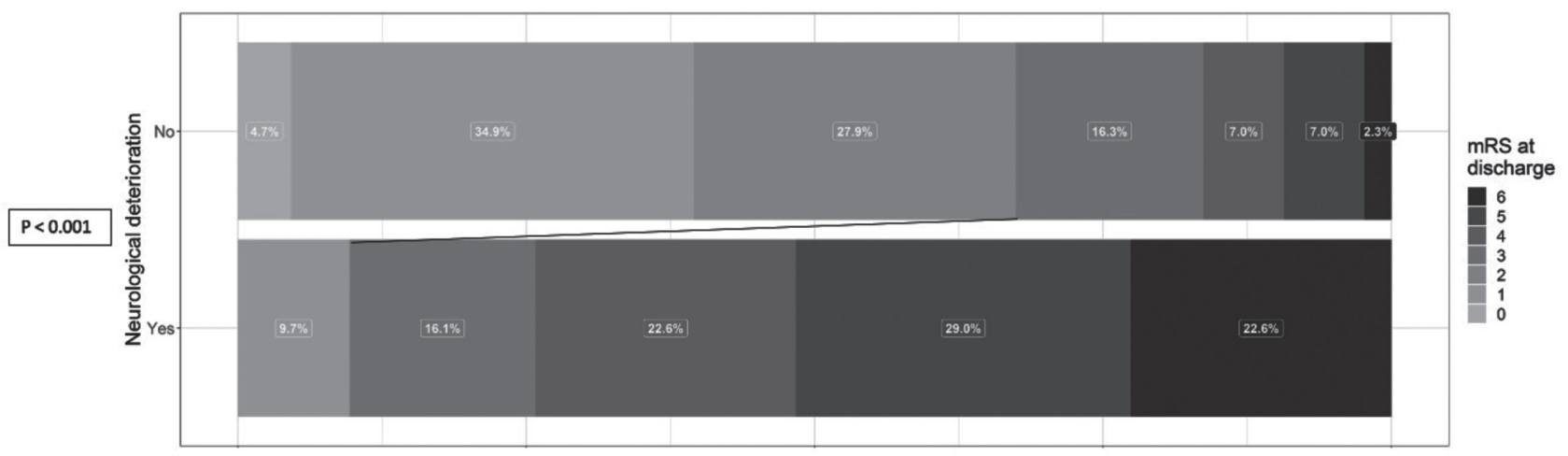

Figure 2 Comparison of modified Rankin scores at discharge date between cerebellar stroke patients with and without neurological deterioration

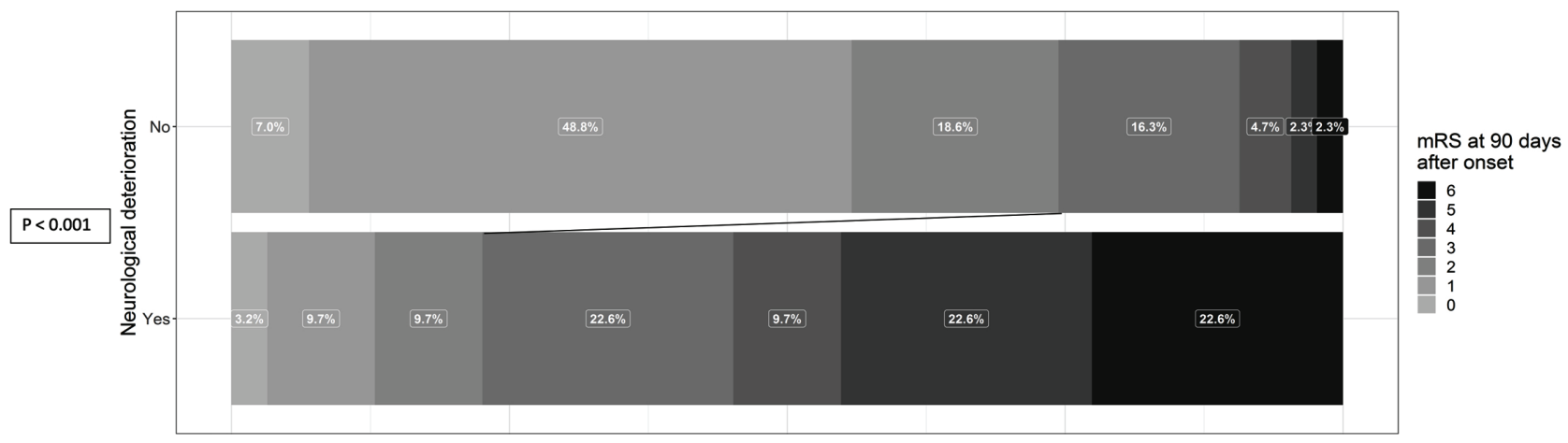

Figure 3 Comparison of modified Rankin scores at 90 days after onset between cerebellar stroke patients with and without neurological deterioration 
The shorter time from stroke onset to hospital arrival and hemispheric cerebellar signs at presentation were protective factors on multivariate analysis (Table 3 ). We considered that the presence of cerebellar hemispheric signs was well realized by most physicians as having a hemispheric cerebellar disorder: facilitating immediate neuro-imaging study and management. In contrast, in cases of cerebellar vermis stroke, they are mostly under evaluated, or missed as peripheral vestibular disorders causing delayed diagnosis and proper management. As found in our study, the presence of hemispheric cerebellar signs was a significant protective factor for NDDA (Table 3). Positive hemispheric cerebellar signs, corresponding with the presence of hemispheric cerebellar lesions seen on the imaging studies, were $50 / 74(68.0 \%)$ cases in our study. From this, 34/43 of patients with positive hemispheric cerebellar signs $(79.0 \%)$ acquired a favorable outcome; eventually. A study by Erik et al. supported our findings, in that cerebellar vermis hemorrhage was associated with higher rates of neurological deterioration due to early, direct compression of the hematoma against the brainstem. ${ }^{6}$

Our study found significantly higher proportions of headaches as a presenting symptom, and elevated DBP in $\mathrm{CH}$ patients. Moreover, white blood cells $\geq 10,000$ cells/ $\mathrm{mm}^{3}$, obstructive hydrocephalus, and distortion of the fourth ventricle on initial brain scans were significantly more prevalent in $\mathrm{CH}$ as well (Table 2). We propose that an abrupt and rapid increment of posterior cranial fossa pressure from cerebellar hematoma; plus its later expansion, possibly causes the anatomical distortions. Furthermore, acute physiological reaction to the acutely elevated intracranial pressure (ICP) probably results in leukocytosis. A study by Furlan showed that leukocytosis on admission was associated with poor outcome too. ${ }^{17}$

Notably, we found no significant difference in NDDA between the two subtypes of cerebellar strokes. In addition, $\mathrm{CH}$ was not an independent risk factor of NDDA by logistic regression analysis in the current study (Table 3). However, the time from onset to NDDA was significantly shorter in $\mathrm{CH}$ (Table 2). This is explainable by more rapid rising of ICP in $\mathrm{CH}$ than in $\mathrm{Cl}$. Unlike a few previous studies ${ }^{6,8}$, which included a wider range of severe cerebellar stroke cases, the significant differences in characteristics between $\mathrm{CH}$ and $\mathrm{Cl}$ were more obvious than ours. As we aimed to determine the predictors of NDDA in the patients initially without indication for neurosurgical interventions, we excluded all the cases with neurological deterioration at their first presentations. And, the limited number of cases enrolled in our study was likely to have fewer clinical parameters with statistically significant differences reported.

Hyperglycemia (Blood sugar $140 \mathrm{mg} / \mathrm{dL}$ ) was not an independent predictor of NDDA in the current study. (Table 3) Actually, we found that the median (IQR) blood sugar (BS) level in our cases $(117(100,133.7) \mathrm{mg} / \mathrm{dL})$ was lower than some previous studies (>150 mg/dL). ${ }^{7,8,18}$ Therefore, lower BS level possibly contributes to better cerebellar stroke outcomes in our study. To our knowledge, hyperglycemia worsens the overall stroke outcomes, because high blood sugar level has been known to exert adverse effects on the structures of cerebral vascular endothelial cells, and to induce acute oxidative stress along with vascular endothelial inflammation. ${ }^{19,20}$

$\mathrm{Koh}^{1}$ concluded that hydrocephalus, brain stem deformity and basal cistern compression were associated with NDDA in cerebellar infarction. St. Louis ${ }^{6}$ also reported that patients with a cerebellar vermis hematoma and acute hydrocephalus were at high risk for NDDA. Furthermore, $\mathrm{Ho}^{8}$ reported that obliteration of basal cistern on the initial CT brain scans was associated with NDDA in cerebellar hemorrhages. Based on our available results, we found no neuro-imaging abnormality as a predictor of NDDA by multivariate analysis (Table 3 ). Since most of the brain images done in our study were CT brain scans, demonstration of such mentioned imaging abnormalities in association with NDDA is possibly obscured. 
Thirty-one $(42.0 \%)$ of all cerebellar stroke patients developed NDDA, and 28 of them (90.0\%) acquired unfavorable neurological outcomes at hospital discharge. When compare with the overall cerebellar stroke outcomes, only $37.8 \%$ had unfavorable outcomes at discharge (2.4 folds higher number in NDDA cases) (Table 1). Some previous studies reported a slightly higher percentage of unfavorable final outcomes $(50.0 \%) .{ }^{1,6,8}$ With the available information and based on our current findings, a worse neurological outcome is undoubtedly higher in NDDA cerebellar stroke patients.

Under limitation of accessibility of MRI brain, particularly under emergency service setting in our center and the similar others, we speculated that some clinical presentation characteristics could be practically useful to predict the occurrence of NDDA among the initially nonsurgical-indicated patients. We expect that our findings could facilitate appropriate monitoring and timely starting of necessary neurosurgical interventions aiming at favorable cerebellar stroke outcomes.

The limitations of the current study are retrospective design and single-center study with limited sample size. Further prospective and multi-center studies, which include more study samples with variability of cerebellar stroke severity will be useful in providing an appropriate management decision on initiation of early neurosurgical interventions for cerebellar stroke patients.

\section{Conclusion}

Cerebellar hemorrhage was not an independent predictor for NDDA for all cerebellar strokes in this study. Some initial clinical presentations of cerebellar stroke, regardless of types of cerebellar stroke, are potentially applicable to predict NDDA along with favorable short and long-term outcomes.

\section{Acknowledgement}

The authors thank all the contributions of all patients as well as the attending physicians of the Department of Internal Medicine and Department of Neurosurgery, Faculty of Medicine, Prince of Songkla University. We thank Miss Nannapat Pruphetkaew for her assistance in data analysis, and also Mr. Andrew Jonathan Tait for editing of the English writing for this manuscript.

\section{Funding sources}

There was no funding or grant received for this research study.

\section{Conflict of interest}

The authors report no conflicts of interest.

\section{References}

1. Koh MG, Phan TG, Atkinson JLD, Wijdicks EFM. Neuroimaging in deteriorating patients with cerebellar infarcts and mass effect. Stroke 2000;31:2062-7.

2. Tohgi H, Takahashi S, Chiba K, Hirata Y. Cerebellar infarction clinical and neuroimaging analysis in 293 patients. Stroke 1993;24:1697-701.

3. Amar AP. Controversies in the neurosurgical management of cerebellar hemorrhage and infarction. Neurosurg Focus 2012; 32:E1.

4. Kase CS, Norrving B, Levine SR, Babikian VL, Chodosh EH, Wolf PA, et al. Cerebellar infarction clinical and anatomic observations in 66 cases. Stroke 1993;24:76-83.

5. Jauss M, Krieger D, Hornig C, Schramm J, Busse O. Surgical and medical management of patients with massive cerebellar infarctions: results of the German-Austrian cerebellar infarction study. J Neurol 1999;246:257-64.

6. St. Louis EK, Wijdicks EFM, Li H. Predicting neurologic deterioration in patients with cerebellar hematomas. Neurology 1998:51:1364-9.

7. Wu YT, Li TY, Lu SC, Chen LC, Chu HY, Chiang SL, et al. Hyperglycemia as a predictor of poor outcome at discharge in patients with acute spontaneous cerebellar hemorrhage. Cerebellum 2012;11:543-8. 
8. Ho YH, Hsu SY, Lin YT, Cheng FC, Lin YJ, Tsai NW, et al. Predictive factors of neurologic deterioration in patients with spontaneous cerebellar hemorrhage: a retrospective analysis. BMC Neurology 2019;19:81.

9. Cano LM, Cardona P, Quesada H, Mora P, Rubio F. Cerebellar infarction: Prognosis and complications of vascular territories. Neurologia 2012;27:330-5.

10. Pong V, Chan KH, Chong BH, Lui WM, Leung GKK, Tse HF, et al. Long-term outcome and prognostic factors after spontaneous cerebellar hemorrhage. Cerebellum 2012;11:939-45.

11. Powers WJ, Rabinstein AA, Ackerson T, Adeoye OM, Bambakidis NC, Becker K, et al. 2018 guidelines for the early management of patients with acute ischemic stroke. Stroke 2018;49:e4699.

12. Anderson CS, Heeley E, Huang Y, Wang J, Stapf C, Delcourt $C$, et al. Rapid blood-pressure lowering in patients with acute intracerebral hemorrhage. N Engl J Med 2013;368: 2355-65.

13. Qureshi Al, Palesch YY, Barsan WG, Hanley DF, Hsu CY, Martin RL, et al. Intensive blood-pressure lowering in patients with acute cerebral hemorrhage. N Engl J Med 2016;375: 1033-43.

14. Hemphill JC, Greenberg SM, Anderson CS, Becker K, Bendok BR, Cushman M, et al. Guidelines for the management of spontaneous intracerebral hemorrhage. Stroke 2015;46: 2032-60.

15. Zhang Y, Reilly KH, Tong W, Xu T, Chen J, Bazzano LA, et al. Blood pressure and clinical outcome among patients with acute stroke in Inner Mongolia, China. J Hypertens 2008;26: 1446-52.

16. Rodriguez-Luna D, Pineiro S, Rubiera M, Ribo M, Coscojuela $\mathrm{P}$, Pagola $\mathrm{J}$, et al. Impact of blood pressure changes and course on hematoma growth in acute intracerebral hemorrhage. Eur J Neurol 2013;20:1277-83.

17. Furlan JC, Vergouwen MDI, Fang J, Silver FL. White blood cell count is an independent predictor of outcomes after acute ischaemic stroke. Eur J Neurol 2014;21:215-2.

18. Tao C, Hu X, Wang J, You C. Effect of admission hyperglycemia on 6-month functional outcome in patients with spontaneous cerebellar hemorrhage. Med Sci Monit 2017;23: 1200-7.

19. Dandona P, Chaudhuri A, Ghanim H, Mohanty P. Proinflammatory effects of glucose And anti-inflammatory effect of insulin: relevance to cardiovasculardisease. Am J Cardiol 2007;99(Suppl):15B-26B.

20. Lindsberg PJ, Roine RO. Hyperglycemia in acute stroke. Stroke 2004;35:363-4. 